\title{
Considerações biomecânicas de próteses implantossuportadas parafusadas e cimentadas
}

\author{
Biomechanical considerations screw and cemented implant prostheses
}

TORO, Carmen Victoria Torres ${ }^{1}$; VALENTE, Mariana Lima da Costa $^{2}$; REIS, Andréa Candido dos ${ }^{3}$

1. Doutora em Odontologia Restauradora, Faculdade de Odontologia de Ribeirão Preto, Universidade de São Paulo, Ribeirão Preto, SP, Brasil.
2. Doutoranda em Reabilitação Oral, Faculdade de Odontologia de Ribeirão Preto, Universidade de São Paulo, Ribeirão Preto, SP, Brasil.
3.Professora Associada do Departamento de Materiais Dentários e Prótese, Faculdade de Odontologia de Ribeirão Preto, Universidade de São Paulo, Ribeirão Preto, SP, Brasil.

Endereço para correspondência:

Andréa Cândido dos Reis

Endereço: Av. do Café, s/n ${ }^{\circ}$

14040-904 - Ribeirão Preto - São Paulo - Brasil

E-mail: andreare73@yahoo.com.br

Recebido: 31.08.2016

Aceito: 30.06 .2017

\section{RESUMO}

O presente estudo teve por objetivo apresentar por meio de uma revisão da literatura diferentes critérios envolvidos na seleção do sistema de retenção de próteses sobre implantes, a fim de facilitar a escolha do cirurgião-dentista. Para isso foi realizada busca no PubMed utilizando-se os termos - "dental implants", "cemented-retained prosthesis", "screwed-retained prosthesis", "biomechanical implant prosthesis". Os artigos de maior relevância para o tema, escritos em inglês e publicados entre 1986 e 2016 foram selecionados. Na literatura existem vários estudos longitudinais, clínicos e laboratoriais abordando reabilitações parciais ou totais com implantes odontológicos. O sistema de retenção das próteses, cimentada ou parafusada, apresenta vantagens e desvantagens e, apesar de não interferir no sucesso do tratamento, a seleção do método mais adequado ainda representa grande dificuldade entre os profissionais da odontologia, principalmente porque envolve uma série de fatores como passividade, reversibilidade, oclusão, estética. A escolha da modalidade de retenção das próteses sobre implantes deve ser realizada por meio de um diagnóstico e plano de tratamento prévio, avaliando-se individualmente as características do paciente, suas expectativas e as vantagens e desvantagens de cada um dos sistemas.

Palavras-chave: Implantes dentários. Próteses e implantes. Retenção em prótese dentária.

\section{ABSTRACT}

The present study aimed to intorduce through a literature review different criteria involved in the selection denture retention system of implants in order to facilitate the choice of dentist. For this search was conducted in PubMed using the terms - "dental implants", "cemented-retained prosthesis," "screwed-retained prosthesis," "biomechanical implant prosthesis." The most relevant articles to the topic, written in English and published between 1986 and 2016 were selected. In the literature there are several longitudinal, clinical and laboratory studies addressing partial or total rehabilitation with dental implants. The retention system of the prosthesis, cemented or screwed, has advantages and disadvantages and, despite not interfere with successful treatment, the selection of the most appropriate method still represents great difficulty among dental professionals, especially because it involves a number of factors such as passivity, reversibility, occlusion, aesthetics. The choice of the prosthetic implant retention mode should be performed by a prior diagnosis and treatment planning, assessing individual patient characteristics, their expectations and the advantages and disadvantages of each system.

Keywords: Dental implants. Prostheses and implants. Dental prosthesis retention. 


\section{INTRODUÇÃO}

O aumento da expectativa de vida da população, aliado à maior exigência estética dos pacientes e aos ótimos resultados biológicos, clínicos, funcionais e estéticos das reabilitações parciais ou totais com implantes dentais, tem tornado esta, uma alternativa de sucesso para alcançar bons resultados protéticos ${ }^{1-2}$, sendo atualmente uma das principais escolhas entre os pacientes.

A união da prótese ao pilar protético ainda é um dos pontos críticos para alcançar, a longo prazo, a durabilidade dessa terapia, que pode ocorrer por meio de aparafusamento, cimentação ou uma combinação de ambos ${ }^{3}$. Cada modalidade possui vantagens e desvantagens $^{4-5}$, as próteses parafusadas apresentam um histórico favorável em pacientes edêntulos ${ }^{6}$, porém, a falta de ajuste passivo das mesmas leva a uma maior concentração de tensões ao redor do implante. As cimentadas, promovem oclusão mais eficiente, estética favorável e menor intensidade de carga ${ }^{7}$. Declarações da $3^{\mathrm{a}}$ Conferência de Consenso da Associação Europeia de Osseointegração concluíram que ambos os tipos de reconstruções influenciam os resultados clínicos, mas nenhum dos métodos de fixação é claramente vantajoso em relação ao outro ${ }^{8}$.

Embora as reabilitações protéticas com implantes apresentem um elevado índice de sucesso, complicações biológicas e técnicas são frequentes5. Dentre os problemas mais citados está o mecânico, que pode ser afetado por diversas razões como planejamento inadequado, material, condições locais e sistêmicas, hábitos parafuncionais, design da prótese e manutenção da mesma ${ }^{9-12}$.

A fim de garantir uma biomecânica satisfatória, a seleção do sistema de retenção da prótese implantossuportada deve ser realizada previamente a etapa cirúrgica, durante a fase de planejamento, com o objetivo de determinar o posicionamento e angulação mais adequados do implante no leito ósseo, além do espaço suficiente para a prótese $^{12}$. Aliado a isso, a avaliação de fatores como a passividade na adaptação, planejamento oclusal e estético, preservação da saúde dos tecidos moles peri-implantares e reversibilidade são fatores imprescindíveis para a escolha da restauração protética mais adequada.

A oclusão do paciente também consiste um importante fator para a biomecânica e sua avaliação é indispensável para a correta seleção da prótese, visto que está diretamente relacionada a distribuição de cargas aos componentes protéticos e a interface osso/implante ${ }^{13}$. Apesar das conhecidas vantagens e desvantagens das próteses parafusadas e cimentadas, os profissionais ainda apresentam dúvidas no momento da indicação de uma ou outra modalidade de tratamento frente aos casos clínicos. Assim, o objetivo deste estudo foi avaliar aspectos prioritários na seleção do sistema de retenção de próteses implantossuportadas como forma de facilitar a escolha do protesista.

\section{REVISÃO DE LITERATURA}

\section{Adaptação - Passividade}

Ao contrário dos dentes naturais, os implantes não possuem mobilidade fisiológica, proporcionada pelo ligamento periodontal, essencial para a distribuição das forças ao longo do comprimento da raiz e capaz de compensar pequenas distorções que ocorrem durante o processo de confecção da prótese ${ }^{14-15}$. Assim, a passividade é um fator indispensável para uma boa adaptação e dissipação de tensões, prevenir a indução de cargas lesivas no tecido ósseo, no implante6, ${ }^{16-17}$ e garantir a longevidade das próteses implantossuportadas ${ }^{18-20}$.

A falta de passividade da infraestrutura da prótese implanto-suportada tem sido citada como um dos principais elementos relacionados às complicações biológicas e falhas mecânicas dos componentes do sistema prótese/implante ${ }^{21-22}$. As biológicas estão associadas ao aumento da transferência de cargas para o tecido ósseo, implante, formação de gaps entre o pilar e a prótese, além de consequente reabsorção óssea, devido ao acúmulo de micro-organismos nessa região ${ }^{6,17}$. As mecânicas referemse principalmente ao afrouxamento ou fratura do parafuso de fixação, fratura do implante, da infraestrutura da prótese e da porcelana ${ }^{6,17,23}$

Diversos fatores, em diferentes estágios da confecção da prótese ${ }^{6}$ podem levar a ausência de passividade como na moldagem, obtenção do modelo mestre, confecção dos padrões de cera, inclusão, fundição, queima da porcelana ou instalação da prótese. Alguns procedimentos podem ser realizados a fim de minimizar as discrepâncias, como utilizar materiais de moldagem apropriados, com baixa deformação elástica e avaliar radiograficamente a adaptação dos transferentes ${ }^{24-25}$. Mas, ainda hoje as condutas clínicas e laboratoriais empregadas na fabricação de estruturas são inadequadas para oferecer um ajuste completamente passivo das próteses implantossuportadas, principalmente das parafusadas.

Uma situação de perfeita passividade é difícil de ser obtida, entretanto, os componentes protéticos e o tecido ósseo peri-implantar são capazes de tolerar certo grau de desadaptação sem gerar problemas biomecânicos ${ }^{15-16}$. Alguns estudos mostram que as próteses cimentadas apresentam maior passividade ${ }^{13,18,20}$, devido ao espaço interno para ocupação do cimento ${ }^{17,26-27}$ e a adaptação da 
prótese ao pilar protético pré-fabricado ou sobrefundido, diferente do que ocorre nas parafusadas, onde o pilar e o coping constituem uma única peça. Outro fator prejudicial para passividade das próteses parafusadas é a força aplicada ao parafuso, que pode introduzir tensões na interface prótese/implante ${ }^{13,18,20,28-29}$.

\section{Oclusão}

A escolha do tipo de retenção da prótese sobre implante exerce grande influência na oclusão do paciente. Nas restaurações cimentadas, contatos oclusais ideais e estáveis podem ser estabelecidos possibilitando a transmissão das forças ao longo eixo do sistema prótese/implante ${ }^{20,29}$. As parafusadas, por apresentarem orifício de acesso na região oclusal podem interferir nos contatos oclusais cêntricos e nos movimentos excursivos de protrusão e lateralidade ${ }^{13,19}$.

Os materiais utilizados para cobrir o orifício de acesso, que ocupa cerca de $50 \%$ da superfície oclusal em molares e $75 \%$ em pré-molares, são suscetíveis ao desgaste sob forças mastigatórias, diminuindo a preservação dos contatos funcionais e comprometendo a durabilidade da restauração ${ }^{17}$. Ainda, a presença do orifício de entrada do parafuso, pode aumentar o risco de fratura da porcelana, possivelmente devido à ausência de infraestrutura subjacente na área ${ }^{13,17,23,27,30}$.

A dificuldade em conseguir contatos oclusais estáveis ao utilizar próteses parafusadas afeta a direção da distribuição de cargas, gerando forças laterais ao implante, fenômeno que não é observado nas cimentadas visto que apresentam superfície oclusal intacta ${ }^{3}$.

\section{Estética}

Quando o planejamento é realizado de forma adequada e os implantes são instalados em posição ideal, resultados estéticos previsíveis podem ser alcançados com qualquer tipo de restauração, cimentada ou parafusada. No entanto, quando o implante apresenta inclinação desfavorável e o parafuso de acesso fica visível na zona estética a prótese de escolha, baseada neste requisito é a cimentada ${ }^{16-17,31}$.

Isso porque, apesar dos diversos recursos estéticos presentes atualmente na odontologia, ainda há grande dificuldade na mimetização da porcelana e resina, ou seja, nestes casos a continuidade da escultura oclusal ou palatina é interrompida e a cor do parafuso fica visíve $e^{16,16,31-32}$.

Quando a instalação dos implantes em posição ideal não é possível, devido à presença de alguma limitação anatômica, pilares angulados ou personalizados podem ser utilizados para que o orifício de acesso fique localizado longe da área estética ${ }^{3}$. Porém, nestes casos também é preciso levar em conta a presença da cinta metálica dos componentes, outro fator prejudicial em locais onde a estética é preponderante.

Nas próteses cimentadas também é possível utilizar pilares personalizáveis para corrigir o mau posicionamento dos implantes e, ao contrário das restaurações parafusadas esse método apresenta como vantagens a reprodução do contorno sinuoso e a uniformidade da profundidade do sulco gengival, em especial quando o contorno é irregular ${ }^{33-34}$.

\section{Adaptação Marginal e Tecidos Peri-implantares}

O tecido gengival sadio e a mucosa peri-implantar apresentam infiltrados inflamatórios com localização e extensão similares entre si, porém, a ausência do cemento radicular na superfície dos implantes altera o plano de orientação e união das fibras, tornando o tecido gengival ao redor dos mesmos mais susceptível às inflamações ${ }^{35}$.

Com relação ao tipo de retenção, as parafusadas apresentam discrepância marginal entre o pilar e a prótese menor em comparação as cimentadas, o que permite uma melhor adaptação marginal ${ }^{18,36}$. Além disso, a presença de cimento na região subgengival associada à dificuldade de remoção do mesmo ${ }^{37}$, pode levar a inflamações e doenças peri-implantares como a mucosite peri-implantar e periimplantite ${ }^{37-40}$, especialmente quando a margem subgengival da prótese é maior que $3 \mathrm{~mm} 3,{ }^{17}$.

A discrepância marginal das próteses cimentadas varia segundo o tipo de cimento utilizado ${ }^{36}$, quando ele é temporário, há uma maior facilidade de dissolução do mesmo, o que pode ocasionar maior retenção de alimentos, placa bacteriana e cálculo, aumentando o risco de periimplantite $^{6,41}$.

Apesar da resposta gengival ser considerada melhor com o uso de próteses parafusadas, devido à ausência de cimento, o afrouxamento do parafuso de fixação pode levar a formação de tecido de granulação, fístulas, deposição de placa bacteriana e fratura do parafuso na interface prótese/pilar ou implante pilar ${ }^{3,42}$.

Para evitar essas complicações recomenda-se que os pacientes voltem uma semana após a instalação das próteses para verificar a existência de resíduos de cimento no caso das próteses cimentadas ${ }^{6}$ e para reapertar os parafusos de fixação no caso das parafusadas, de 5 anos em 5 anos ${ }^{43}$.

Estudos realizados a longo prazo, não apresentam consenso a respeito de qual seria o melhor tipo de sistema de retenção para os tecidos marginais. Alguns mostram que as próteses parafusadas proporcionam uma gengiva mais saudável, com menores complicações biológicas ${ }^{44-46}$, outros observam que as cimentadas apresentam melhor resposta biológica gengival e óssea ${ }^{23}$. Existem estudos ainda, que não encontram diferença no comportamento do tecido ósseo marginal e tecido mole peri-implantar em ambos os tipos de retenção ${ }^{47-48}$.

\section{Retenção}

Os fatores que influenciam a retenção das próteses cimentadas, rotineiramente utilizadas na odontologia, 
assemelham-se muito aos envolvidos em procedimentos de próteses convencionais. A oclusão favorável, custo reduzido, menor complexidade dos componentes protéticos e procedimentos laboratoriais são alguns dos benefícios dessa forma de retenção? ${ }^{7}$.

Enquanto nos dentes naturais o principal fator responsável pela retenção das coroas é a convergência das paredes axiais ${ }^{6,13}$, nas próteses sobre implantes cimentadas, as características morfológicas, a área de superfície, altura e aspereza superficial dos pilares possibilitam retenção três vezes maior se comparada à obtida em dentes naturais.

Outro importante requisito envolvido na retenção das próteses sobre implantes cimentadas é a escolha do agente cimentante. A maioria dos cimentos utilizados em odontologia foram desenvolvidos para uso em dentes naturais, dessa forma, no caso dos implantes, a ação dos mesmos ocorre de maneira diferente, visto que devem se opor ao deslocamento de duas superfícies metálicas, contrário ao que normalmente ocorre em próteses convencionais ${ }^{49}$.

Dessa forma, a seleção do cimento deve ser criteriosa e considerar importantes fatores como as especificações do pilar e da coroa, as características das superfícies opostas, o grau de retenção desejado e as propriedades específicas de cada agente de cimentação $0^{49-51}$.

Apesar dos benefícios notáveis das próteses cimentandas, como simplificação dos procedimentos restauradores, redução dos custos, passividade e ausência de aberturas de acesso ao implante, a reduzida taxa de reversibilidade e a presença de cimento residual, responsável na maioria das vezes pelos processos inflamatórios peri-implantares são algumas das desvantagens desses sistemas ${ }^{52}$.

Nas próteses parafusadas, a retenção ocorre por meio de parafusos que realizam a conexão entre o implante, $o$ pilar e a prótese, como consequência à resistência friccional presente entre as roscas internas do implante e as do parafuso de fixação ${ }^{6}$. Neste caso, ao contrário do que ocorre nas cimentadas, onde a altura das paredes axiais é um fator decisivo, o espaço interoclusal reduzido não representa um fator limitante para sua escolha ${ }^{16-17,53}$.

Ao longo do tempo, pode ocorrer o afrouxamento do parafuso de fixação ( 3 a $6 \%$ ) e este, quando não detectado pelo paciente, pode levar a complicações como sobrecarga biomecânica, aplicação de forças fora do longo eixo e desajuste dos componentes e prótese, afetando a quantidade de retenção da restauração ${ }^{3,44,54-55}$. Para alcançar força de aperto suficiente deve-se seguir as especificações dos fabricantes e utilizar chaves de controle de torque, além disso, deve-se realizar o retorque 5 minutos após o inicial e algumas semanas após de acordo com o recomendado ${ }^{56}$.

Embora a principal vantagem das próteses parafusadas seja a reversibilidade, simplificando a higiene das superestruturas, reparos e o aperto dos parafusos de fixação; a presença dos orifícios na superfície da restauração, pode comprometer a oclusão, estética e biomecânica das mesmas ${ }^{52}$.

Diante disso, pode-se observar que as vantagens e desvantagens de cada sistema, por si só, não são suficientes para garantir o sucesso da restauração protética, sendo extremamente importante levar em consideração diversos aspectos na seleção do sistema de retenção, desde a biomecânica até a condição clínica do paciente ${ }^{57-58}$.

\section{Reversibilidade}

A reversibilidade das próteses sobre implantes constitui um importante fator, visto que possibilita a substituição ou recuperação das mesmas em caso de necessidade de troca dos componentes protéticos, afrouxamento ou fratura dos parafusos de fixação e pilares, alteração da prótese após a perda de um implante, intervenção cirúrgica e remodelamento estético ${ }^{3,6,13,44}$.

As próteses parafusadas apresentam como principal vantagem, a reversibilidade, o que evita danos na restauração e no sistema de fixação quando da necessidade de reparos $^{17}$ e facilita as sessões clínicas de controle ${ }^{11}$. Embora as próteses cimentadas simplifiquem o planejamento e a reabilitação final, apresentam como importante desvantagem a dificuldade potencial de recuperação da restauração, geralmente fraturada durante $o$ procedimento de remoção, além de causar possíveis danos às roscas internas do implante devido a aplicação de forças sobre a coroa solta ${ }^{58}$.

Vários estudos têm relatado técnicas para a recuperação de próteses cimentadas com danos mínimos, como a utilização de cimentos provisórios para a cimentação de peças definitivas ou ainda o desenvolvimento de dispositivos que auxiliem na remoção das mesmas ${ }^{57-59}$. Enquanto outros autores consideram esses procedimentos difíceis e na maioria das vezes inviáveis ${ }^{29}$, sem contar que não apresentam resultados previsíveis a longo prazo $^{60-61}$.

\section{Provisionalização}

A fim de manter excelente perfil estético dos tecidos moles ao redor das restaurações implantossuportadas, autores $^{62-63}$ têm optado recentemente pelo carregamento imediato dos implantes com próteses provisórias ${ }^{64-65} \mathrm{e}$ relatam taxa de sobrevivência de $100 \%$ com perda mínima da crista óssea.

Alguns autores ${ }^{16,66}$ preferem a utilização das próteses parafusadas, porque o parafuso de fixação pode ser utilizado para expandir e modelar a mucosa peri-implantar de acordo com a força aplicada e relatam como principal desvantagem associada às provisórias cimentadas, a dificuldade na remoção do excesso de cimento, possível inflamação na margem gengival causada pelo mesmo e a contenção da hemorragia ${ }^{3,16}$. 


\section{DISCUSSÃO}

Em geral, é durante a fase de planejamento ${ }^{67}$ que o cirurgião dentista deve selecionar o método de retenção das próteses sobre implantes, parafusado ou cimentado, tendo em vista que a literatura apresenta diferentes vantagens e desvantagens de cada um desses sistemas ${ }^{3,5-6,17}$.

As próteses parafusadas apresentam vantagens como reversibilidade, necessidade de espaço interoclusal mínimo, fácil remoção para manutenção, reparos ou intervenções cirúrgicas. Por ouro lado, exigem posicionamento ideal do implante, devido a presença do orifício de acesso e técnica mais sensível que as cimentadas6, que possuem custo mais acessível, facilidade na compensação da inclinação de implantes, passividade, estética e oclusão favoráveis ${ }^{5,13,40}$. O principal problema associado a cimentação dessas restaurações é a dificuldade na remoção do excesso de cimento, que pode levar a complicações biológicas como doenças peri-implantares ${ }^{37-39}$.

Devido às informações conflitantes na literatura, o clínico apresenta grande dificuldade no momento da escolha do tipo de retenção ${ }^{11,44,68-69}$. Enquanto alguns estudos $^{23}$ demonstram que as restaurações cimentadas são superiores às parafusadas clínica e biologicamente, devido à maior passividade ${ }^{19,70}$, estética e oclusão, outros relatam que as coroas parafusadas, por serem reversíveis, são necessárias na presença de vários pilares ${ }^{16-17} \mathrm{e}$ ainda existem aqueles que não demostram diferença significativa entre um e outro sistema no que diz respeito às altas taxas de sobrevivência dos implantes ${ }^{63,67,71}$.

Baseando-se nos achados encontrados na literatura podemos verificar que não existe uma padronização entre os autores na indicação de um sistema de retenção ideal para próteses implantossuportadas. A seleção do mesmo irá depender de uma série de fatores clínicos, laboratoriais e técnicos, cabendo ao profissional realizar um diagnóstico e planejamento completos considerando as características e expectativas de cada paciente quanto ao tratamento, a fim de determinar qual o melhor método de retenção para cada caso clínico específico.

\section{CONCLUSÃO}

A literatura é composta por diversos estudos que identificam vantagens e desvantagens das próteses sobre implantes cimentadas e parafusadas, mas não apresenta uma padronização na indicação de uma ou outra modalidade de tratamento. Assim, cabe ao profissional de odontologia conhecer as limitações de cada sistema e a seleção do método mais adequado frente a um caso clínico.

\section{REFERÊNCIAS}

1. da Costa Valente ML, de Castro DT, Shimano AC, Lepri CP, dos Reis AC. Analyzing the influence of a new dental implant design on primary stability. Clin Implant Dent Relat Res. 2016;18(1):168-73.

2. Yunus N, Masood M, Saub R, Al-Hashedi AA, Taiyeb Ali TB, Thomason JM. Impact of mandibular implant prostheses on the oral health-related quality of life in partially and completely edentulous patients. Clin Oral Implants Res. 2016;27(7):904-9.

3. Shadid R, Sadaqa N. A comparison between screw- and cementretained implant prostheses. A literature review. J Oral Implantol. 2012;38(3):298-307.

4. Gervais MJ, Wilson PR. A rationale for retrievability of fixed, implant-supported prostheses: a complication-based analysis. Int J Prosthodont. 2007;20(1):13-24.

5. Wittneben JG, Millen C, Bragger U. Clinical performance of screwversus cement-retained fixed implant-supported reconstructions - a systematic review. Int J Oral Maxillofac Implants. 2014;29:84-98.

6. Michalakis KX, Hirayama H, Garefis PD. Cement-retained versus screw-retained implant restorations: a critical review. Int J Oral Maxillofac Implants. 2003;18(5):719-28.

7. Kapoor R, Singh K, Kaur S, Arora A. Retention of implant supported metal crowns cemented with different luting agents: a comparative in vitro study. J Clin Diagn Res. 2016;10(4):ZC61-4.

8. Gotfredsen K, Wiskott A. Consensus report - reconstructions on implants. The third EAO Consensus Conference 2012. Clin Oral Implants Res. 2012;23(6):238-41.

9. Aglietta M, Siciliano VI, Zwahlen M, Brägger U, Pjetursson BE, Lang NP, et al. A systematic review of the survival and complication rates of implant supported fixed dental prostheses with cantilever extensions after an observation period of at least 5 years. Clin Oral Implants Res. 2009;20(5):441-51.

10. Jung RE, Pjetursson BE, Glauser R, Zembic A, Zwahlen M, Lang NP. A systematic review of the 5-year survival and complication rates of implant-supported single crowns. Clin Oral Implants Res. 2008;19(2):119-30.

11. Pjetursson BE, Bragger U, Lang NP, Zwahlen M. Comparison of survival and complication rates of tooth-supported fixed dental prostheses (FDPs) and implant-supported FDPs and single crowns (Scs). Clin Oral Implant Res. 2007;18(3):97-113.

12. Thalji G, Bryington M, De Kok IJ, Cooper LF. Prosthodontic management of implant therapy. Dent Clin North Am. 2014;58(1):207-25.

13. Hebel KS, Gajjar RC. Cement-retained versus screw-retained implant restorations: achieving optimal occlusion and esthetics in implant dentistry. J Prosthet Dent. 1997;77(1):28-35.

14. Gulati M, Anand V, Govila V, Jain N, Rastogi P, Bahuguna R, et al. Periodontio-integrated implants: a revolutionary concept. Dent Res J (Isfahan). 2014;11(2):154-62.

15. Isa ZM, Hobkirk JA. The effects of superstructure fit and loading on individual implants units: part 1 . The effects of tightening the gold screws and placement of a superstructure with varying degrees of fit. Eur J Prosthodont Restor Dent. 1995;3(6):247-53. 
16. Chee W, Jivraj S. Screw versus cemented implant supported restorations. Br Dent J. 2006;201(8):501-7.

17. Lee A, Okayasu K, Wang HL. Screw-versus cement-retained implant restorations: current concepts. Implant Dent. 2010;19(1):8-15.

18. Guichet DL, Caputo AA, Choi H, Sorensen JA. Passivity of fit and marginal opening in screw- or cement-retained implant fixed partial denture designs. Int J Oral Maxillofac Implants. 2000;15(2):239-46.

19. Heckmann SM, Karl M, Wichmann MG, Winter W, Graef F, Taylor TD. Cement fixation and screw retention: parameters of passive fit. An in vitro study of three-unit implant-supported fixed partial dentures. Clin Oral Implants Res. 2004;15(4):466-73.

20. Taylor TD, Agar JR, Vogiatzi T. Implant prosthodontics: current perspective and future directions. Int J Oral Maxillofac Implants. 2000;15(1):66-75.

21. Duyck J, Naert I. Influence of prosthesis fit and the effect of a luting system on the prosthetic connection preload: an in vitro study. Int J Prosthodont. 2002;15(4):389-96.

22. Romero GG, Engelmeier R, Powers JM, Canterbury AA. Accuracy of three corrective techniques for implant bar fabrication. J Prosthet Dent. 2000;84(6):602-7

23. Nissan J, Narobai D, Gross O, Ghelfan O, Chaushu G. Long-term outcome of cemented versus screw-retained implant-supported partial restorations. Int J Oral Maxillofac Implants. 2011;26(5):1102-7.

24. Herbst D, Nel JC, Driessen CH, Becker PJ. Evaluation of impression accuracy for osseointegrated implant supported superstructures. J Prosthet Dent. 2000;83(5):555-61

25. Hussaini $\mathrm{S}$, Wong $\mathrm{T}$. One clinical visit a multiple implant restoration master cast fabrication. J Prosthet Dent. 1997;78(6):550-3.

26. Rajan M, Gunaseelan R. Fabrication of a cement and screw-retained implant prosthesis. J Prosthet Dent. 2004;92(6):578-80

27. Zarone F, Sorrentino R, Traini T, Di lorio D, Caputi S.Fracture resistance of implant-supported screw versus cement-retained porcelain fused to metal single crowns: SEM fractographic analysis. Dent Mater. 2007;23(3):296-301

28. Sahin S, Cehreli MC. The significance of passive framework fit in implant prosthodontics: current status. Implant Dent. 2001;10(2):85-92.

29. Taylor TD, Agar JR. Twenty years of progress in implant prosthodontics. J Prosthet Dent. 2002;88(1):89-95.

30. Torrado E, Ercoli C, A1 Mardini M, Graser GN, Tallents RH, Cordaro L. A comparison of the porcelain fracture resistance of screwretained and cement-retained implant-supported metal-ceramic crowns. J Prosthet Dent. 2004;91(6):532-7.

31. Vigolo P, Givani A, Majzoub Z, Cordioli G. Cemented versus screwretained implant-supported single-tooth crowns: A 4-year prospective clinical study. Int $\mathrm{J}$ Oral Maxillofac Implants. 2004;19(2):260-5

32. Weininger B, McGlumphy E, Beck M. Esthetic evaluation of materials used to fill access holes of screw-retained implant crowns. J Oral Implantol. 2008;34(3):145-9.

33. Eger DE, Gunsolley JC, Feldman S. Comparison of angled and standard abutments and their effect on clinical outcomes: a preliminary report. Int J Oral Maxillofac Implants. 2000;15(6):819-23.

34. Sethi A, Kaus T, Sochor P. The use of angulated abutments in implant dentistry: five-year clinical results of an ongoing prospective study. Int J Oral Maxillofac Implants. 2000;15(6):801-10.

35. Lekholm U, Eriksson B, Adell R, Slots J. The condition of the soft tissues at tooth and fixture abutments supporting fixed bridges. A microbiological and histological study. J Clin Periodontol. 1986;13(6):558-62.

36. Keith SE, Miller BH, Woody RD, Higginbottom FL. Marginal discrepancy of screw-retained and cemented metal-ceramic crowns on implants abutments. Int J Oral Maxillofac Implants. 1999;14(3):369-78

37. Wadhwani C, Hess T, Piñeyro A, Opler R, Chung KH. Cement application techniques in luting implant-supported crowns: a quantitative and qualitative survey. Int J Oral Maxillofac Implants. 2012;27(4):859-64.

38. Linkevicius T, Vindasiute E, Puisys A, Linkeviciene L, Maslova N, Puriene A. The influence of the cementation margin position on the amount of undetected cement. A prospective clinical study. Clin Oral Implants Res. 2013;24(1):71-6.

39. Linkevicius T, Vindasiute E, Puisys A, Peciuliene V. The influence of margin location on the amount of undetected cement excess after delivery of cement-retained implant restorations. Clin Oral Implants Res. 2011;22:1379-84.

40. Wilson TG Jr. The positive relationship between excess cement and peri-implant disease: a prospective clinical endoscopic study. J Periodontol. 2009;80(9):1388-92.

41. Agar JR, Cameron SM, Hughbanks JC, Parker MH. Cement removal from restorations luted to titanium abutments with simulated subgingival margins. J Prosthet Dent. 1997;78(1):43-7.

42. Pauletto N, Lahiffe BJ, Walton JN. Complications associated with excess cement around crowns on osseointegrated implants: a clinical report. Int J Oral Maxillofac Implants. 1999;14(6):865-8.

43. Kallus T, Bessing C. Loose gold screws frequently occur in full-arch prostheses supported by osseointegrated implants after 5 years. Int J Oral Maxillofac Implants. 1994;9(2):169-78.

44. Sailer I, Mühlemann S, Zwahlen M, Hämmerle CH, Schneider D. Cemented and screw-retained implant reconstructions: a systematic review of the survival and complication rates. Clin Oral Implants Res. 2012;23(6):163-201.

45. Sherif S, Susarla SM, Hwang JW, Weber HP, Wright RF. Clinician and patient-reported long-term evaluation of screw and cementretained implant restorations: a 5-year prospective study. Clin Oral Investig. 2011;15(6):993-9.

46. Weber HP, Kim DM, Ng MW, Hwang JW, Fiorellini JP. Peri-implant soft-tissue health surrounding cement and screw-retained implant restorations: a multi-center, 3-year prospective study. Clin Oral Implants Res. 2006;17(4):375-9.

47. Cutrim ES, Peruzzo DC, Benatti B. Evaluation of soft tissues around single tooth implants in the anterior maxilla restored with cemented and screw-retained crowns. J Oral Implantol. 2012;38(6):700-5.

48. Vigolo P, Mutinelli S, Givani A, Stellini E. Cemented versus screwretained implant-supported single-tooth crowns: a 10-year randomised controlled trial. Eur J Oral Implantol. 2012;5(4):355-64.

49. Dudley JE, Richards LC, Abbott JR. Retention of cast crown copings cemented to implant abutments. Aust Dent J. 2008;53(4): 332-9. 
50. Alfaro MA, Papazoglou E, McGlumphy EA, Holloway JA. Shortterm retention properties of cements for retrievable implant-supported prostheses. Eur J Prosthodont Restor Dent. 2004;12(1):33-7.

51. Carter GM, Hunter KM, Herbison P. Factors influencing the retention of cemented implant-supported crowns. N Z Dent J. 1997;93(412):36-8.

52. Perea C, Del Río J, Preciado A, Lynch CD, Celemín A, CastilloOyagüe R. Validation of the 'Quality of Life with Implant Prostheses (QoLIP-10)' questionnaire for wearers of cement-retained implantsupported restorations. J Dent. 2015;43(8):1021-31.

53. Drago CJ. Clinical study of the efficancy of gold-tite square abutment screws in cement-retained implant restorations. Int J Oral Maxillofac Implants. 2003;18(2):273-8.

54. Brägger U, Hirt-Steiner S, Schnell N, Schmidlin K, Salvi GE, Pjetursson B, et al. Complication and failure rates of fixed dental prostheses in patients treated for periodontal disease. Clin Oral Implants Res. 2011;22(1):70-7.

55. Cannizzaro G, Leone M, Consolo U, Ferri V, Esposito M. Immediate functional loading of implants placed with flapless surgery versus conventional implants in partially edentulous patients: a 3-year randomized controlled clinical trial. Int J Oral Maxillofac Implants. 2008;23(5):867-75.

56. Cooper LF, Ellner S, Moriarty J, Felton DA, Paquette D, Molina A, et al. Three-year evaluation of single-tooth implants restored 3 weeks after 1 -stage surgery. Int J Oral Maxillofac Implants. 2007;22(5):791-800.

57. McGlumphy EA. Keeping implant screws tight: the solution. J Dent Symp. 1993;1:20-3.

58. Nissan J, Snir D, Rosner O, Kolerman R, Chaushu L, Chaushu G. Reliability of retrievable cemented implant-supported prostheses. J Prosthet Dent. 2016;115(5):587-91.

59. Ichikawa T, Ishida $\mathrm{O}$, Watanabe $\mathrm{M}$, Tomotake $\mathrm{Y}$, Wei H, Jianrong C. A new retrieval system for cement-retained implant superstructures: a technical report. J Prosthodont. 2008;17(6):487-9.

60. Al-Omari WM, Shadid R, Abu-Naba'a L, El Masoud B. Porcelain fracture resistance of screw-retained, and screw-cement-retained implant-supported metal ceramic posterior crowns. J Prosthodont. 2010;19(4):263-73.

61. Wicks R, Shintaku WH, Johnson A. Three-dimensional location of the retaining screw axis for a cemented single tooth implant restoration. J Prosthodont. 2012;21(6):491-3.

62. Cornelini R, Cangini F, Covani U, Wilson TG Jr. Immediate restoration of implants placed into fresh extraction sockets for singletooth replacement: a prospective clinical study. Int J Periodontics Restorative Dent. 2005;25(5):439-47.

63. Crespi R, Capparè P, Gherlone E, Romanos GE. Immediate occlusal loading of implants placed in fresh sockets after tooth extraction. Int $\mathrm{J}$ Oral Maxillofac Implants. 2007;22(6):955-62.

64. Crespi R, Capparè P, Gastaldi G, Gherlone EF. Immediate occlusal loading of full-arch rehabilitations: screw-retained versus cementretained prosthesis. An 8-year clinical evaluation. Int J Oral Maxillofac Implants. 2014;29(6):1406-11.

65. Schneider RL. Fabricating custom provisional restorations for the ITI solid abutment system. J Prosthet Dent. 2002;88(1):105-7.
66. Albrektsson T, Jansson T, Lekholm U. Osseointegrated dental implants. Dent Clin North Am. 1986;30:151-74.

67. Lemos CA, de Souza Batista VE, Almeida DA, Santiago Júnior JF, Verri FR, Pellizzer EP. Evaluation of cement-retained versus screwretained implant-supported restorations for marginal bone loss: a systematic review and meta-analysis. J Prosthet Dent. 2016;115(4):419-27.

68. Belser UC, Mericske-Stern R, Bernard JP, Taylor TD. Prosthetic management of the partially dentate patient with fixed implant restorations. Clin Oral Implants Res. 2000;11(1):126-45.

69. Salvi GE, Bragger U. Mechanical and technical risks in implant therapy. Int J Oral Maxillofac Implants. 2009;24:69-85.

70. Karl M, Taylor TD, Wichmann MG, Heckmann SM. In vivo stress behavior in cemented and screw-retained five-unit implant FPDs. J Prosthodont. 2006;15(1):20-4

71. Sherif S, Susarla HK, Kapos T, Munoz D, Chang BM, Wright RF. A systematic review of screw versus cement-retained implantsupported fixed restorations. J Prosthodont. 2014;23(1):1-9. 